\title{
THE RELIEF OF INTRACTABLE PAIN BY SURGERY
}

\author{
By Murray A. Falconer, M.Ch., F.R.C.S., F.R.A.C.S. \\ Director, Guy's-Maudsley Neurosurgical Unit
}

In general the treatment of any painful condition is the treatment of its cause. But sometimes this is not possible, either because the underlying pathological process cannot be influenced favourably, or because its nature is not known while the pain itself cannot be relieved by medical treatment. Examples of the first subgroup are seen when nerve trunks are involved by malignant disease, by post-radiation fibrosis, or by other forms of scarring, and also the pain of postherpetic neuralgia, of tabes dorsalis and of certain other diseases of the central nervous system. Examples of the second sub-group are seen in tic douloureux and other neuralgias, in causalgia and in painful phantom-limbs. It is in such conditions that, when pain has become severe and disabling, its relief by surgery has to be considered.

\section{Anatomy of the Pain Pathways}

The principle underlying surgical treatment is to section the pain pathways in the nervous system at an appropriate level, and so stop a barrage of abnormal nerve impulses being relayed to the sensorium. As at some levels section of the pain pathways is accompanied by disturbing sideeffects, which may detract from any beneficial result, and as with some conditions a section at one level may succeed whereas a section at another level may fail, knowledge of the pain pathways is essential.

\section{Pain Sensation in the Trunk and Limbs}

Pain impulses from the trunk and limbs are conveyed to the sensorium by three relays of nerve fibres. Those of the first relay are axons of cells in the posterior root ganglion of the spinal nerves, and they include fibres which have ascended in the peripheral nerves from the limbs and the trunk wall, as well as fibres from the sympathetic nervous system that join spinal nerves via the white rami communicantes. These fibres reach the spinal cord in company with afferent fibres conveying other modalities of sensation, and then terminate almost at once in the dorsal gray matter. It is generally taught that, while painful impulses from the viscera pass through the prevertebral sympathetic plexuses, painful impulses from the limbs always pass through the somatic nerves and not through the perivascular sympathetic plexuses. This view, however, is open to doubt, especially in view of the relief of causalgia by sympathectomy.

The second fibre relay is formed by axons of cells in the dorsal gray matter of the spinal cord (substantia gelatinosa of Rolando). These axons quickly cross to the opposite side of the cord and then ascend within the lateral spino-thalamic tract, together with fibres subserving temperature, to the nucleus ventralis posterior of the thalamus

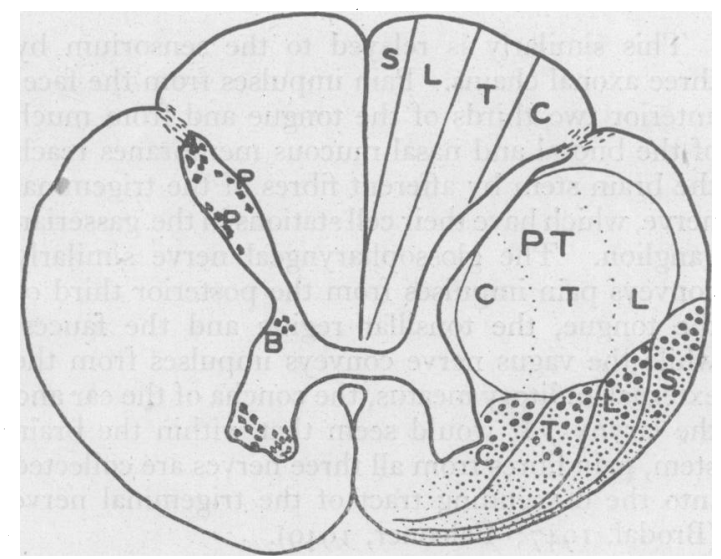

Fig. 1.-The arrangement of the spinothalamic and other tracts in the lower cervical region of the spinal cord, according to A. Earl Walker (1940), Arch. Neurol. Psychiat., Chicago, 43, 284. The heavy dots represent fibres concerned with temperature; medium dots, fibres mediating pain; and fine dots, fibres carrying touch and pressure impulses. Note the lamination of the tracts. $S$ represents fibres conveying impulses from the sacral segments, L lumbar, $T$ thoracic, and C cervical. 
where they terminate. As successive increments of fibres join the lateral spino-thalamic tract, they are arranged on its inner aspect. Thus the tract becomes laminated so that in the cervical cord the fibres conveying impulses from the sacral nerves lie laterally, fibres from the lumbar and thoracic segments are in an intermediate position, while fibres from the cervical segments lie in the medial part of the tract (Fig. I). It would also appear that fibres subserving temperature lie posterior to those subserving pain. Not all pain fibres, however, cross to the opposite side of the cord; a few appear to ascend in the spino-thalamic tract on the same side. Abnormalities of their distribution may occur (French and Peyton, 1948).

The third fibre relay is provided by axons of cells in the nucleus ventralis posterior of the thalamus, which convey impulses to the cerebral cortex, principally to the post-central gyrus, which represents the primary sensory cortical area. There is strong evidence, however, that this is not the only sensory area, for following hemispherectomy pain is still perceived over the opposite side of the body. Recent neurophysiological work suggests that there are secondary sensory areas in other regions of the cerebral cortex for pain impulses from the ipsilateral as well as the contralateral side of the body. There is no certain evidence that pain appreciation can occur in the thalamus (Fulton, 1943; Brodal, 1948).

\section{Pain Sensation in the Head}

This similarly is relayed to the sensorium by three axonal chains. Pain impulses from the face, anterior two-thirds of the tongue and from much of the buccal and nasal mucous membranes reach the brain stem by afferent fibres of the trigeminal nerve, which have their cell stations in the gasserian ganglion. The glossopharyngeal nerve similarly conveys pain impulses from the posterior third of the tongue, the tonsillar region and the fauces, while the vagus nerve conveys impulses from the external auditory meatus, the concha of the ear and the larynx. It would seem that within the brain stem, pain fibres trom all three nerves are collected into the descending tract of the trigeminal nerve (Brodal, 1947; Falconer, 1949).

The second link in the neuronal chain is formed by axons of cells in the nucleus of the descending trigeminal tract. These axons quickly cross over through the brain stem to join the lateral spinothalamic tract in the fillet on the opposite side of the mid-brain, thence they pass upwards to the nucleus ventralis posterior of the thalamus. The axons of cells in this nucleus in their turn supply the third link to the cerebral cortex.
Procedures for the Relief of Pain Arising in the Limbs or Trunk

1. Section of Somatic Nerves-Peripheral Neurectomy

Most somatic nerves contain both motor and sensory fibres, and consequently section of these nerves results in motor paralysis as well as sensory. This greatly limits the scope of peripheral neurectomy, which in practice is generally confined to nerves that are purely sensory or whose motor functions do not matter. Instances are the resection of painful neuromas in ampútation'stumps, of peripheral nerves involved in scars (e.g. an intercostal nerve involved at the site of drainage of an empyema), and division of the lateral cutaneous nerve of the thigh for meralgia paraesthetica. Prior to operation the likely effect of any such nerve section can be judged by blocking the nerve with a local anaesthetic agent.

\section{Section of Sympathetic Pathways-Sympathec- tomy}

Sympathectomy has its greatest rôle in the treatment of post-traumatic pain syndromes, such as the classical causalgia of Weir Mitchell, the minor causalgias of Homans and Livingston, Sudeck's bony atrophy and other forms of traumatic arthritis, and painful amputation stumps. The treatment of these conditions is well surveyed by Livingston (1943), Ulmer and Mayfield (1946), White (1946), Macfarlane (1949), and others. In all these conditions the pain spreads widely and is not confined to the distribution of one nerve; it may be burning (typical of causalgia) or aching, or mixed and it often becomes severe and disabling. In addition these conditions are characterized by vasomotor and trophic disturbances, the extremities showing atrophy of bone and soft parts, and appearing cold, blue and clammy.

It is our experience that sympathectomy, either pre-ganglionic or post-ganglionic, will generally relieve pain in these various conditions, although not in all cases (Macfarlane, 1949). Peripheral neurectomy and posterior rhizotomy are usually ineffective, but chordotomy will almost ahways relieve the pain and can be tried if sympathectomy fails. An indication as to whether sympathectomy will prove effective or not is usually afforded by a procaine block of the appropriate sympathetic pathway. Indeed, sometimes the procaine block will itself be followed by disappearance of the pain for days, weeks, months, or even longer, and in such instances a test performed initially for diagnosis can be repeated at intervals for its therapeutic effect.

Sympathectomy is also of value in relieving pain of visceral origin, but I have had no personal experience in this direction. Its use in angina pec- 
toris has recently been favourably commented upon by Lindgren and Olivecrona (1947) and by White and Bland (1948), and in idiopathic dysmenorrhoea (presacral neurectomy) by Ingersoll and Meigs (1948).

\section{Section of Posterior Nerve Roots-Posterior Rhizotomy}

As a method of relieving pain, posterior rhizotomy has the limitation that it affects all modalities of sensation. When only one posterior nerve root is divided, the resulting sensory loss is scarcely appreciable. Generally, however, a minimum of three successive nerve roots have to be divided (the nerve root almost directly involved and those immediately 'above and immediately below it) in order to ensure that pain-relief is complete. Posterior rhizotomy, therefore, cannot be applied to the treatment of diffuse pain in a limb, because the complete sensory denervation (including postural sensibility) which follows section of several successive roots would render the limb virtually useless. Even an amputation stump would be hampered for the use of a prosthesis. But in the trunk a similar loss of sensibility may not matter much, and posterior rhizotomy, therefore, can be readily applied to conditions in which the segmental nerves of the thoracic or abdominal wall are involved in scar tissue or neoplasm. Posterior rhizotomy, however, will not relieve the pain of causalgia, of painful phantom limbs, nor of postherpetic neuralgia, although it will ease the cutaneous hyperaesthesia which sometimes accompanies these conditions. Attempts have also been made to treat angina pectoris by posterior rhizotomy of the upper five pairs of thoracic nerves, but the results seem somewhat uncertain (Lindgren and Olivecrona, 1947; White and Bland, 1948). Prior to operation the likely effect of posterior rhizotomy in any particular patient can often be ascertained by paravertebral blocking of the appropriate segmental nerves with local anaesthetic agents.

\section{Alcohol Injection of the Cauda Equina}

Since Dogliotti introduced this procedure in I93I, several reports have appeared on the use of intrathecal injections of alcohol for the relief of pain. A satisfactory technique for the cauda equina is outlined by Russell (1936), but the danger of producing a transverse lesion of the spinal cord contraindicates the injection above the first lumbar level. Even for the cauda equina, the results are often unpredictable in individual patients. In a few patients a satisfactory degree of sensory loss is produced without accompanying motor weakness or serious sphincteric disturbance. But often the sensory impairment is patchy or transient, while unless care is taken serious sphincteric disturbances and motor paralyses may be produced. It is, therefore, widely felt that alcohol injection of the cauda equina should be considered only when the patient is a poor risk for operative procedure, or when his life expectancy is less than three months or so because of the malignant nature of his disease. When life expectancy is longer than this, and there are prospects of the patient returning to an active life, chordotomy is preferable.

\section{Section of the Anterolateral Column of the Spinal Cord-Anterolateral Chordotomy}

This ranks first in usefulness among the various operative procedures which are available for relieving pain from the limbs and trunk. It produces loss of sensation only for pain and temperature, the remaining sensory modalities, including touch and posture, being preserved. Often the patient is scarcely aware of any alteration in the sensibility of his body, except when he puts his leg into a bath and finds he cannot tell the temperature. In skilled hands the operation should carry no mortality. For these reasons the procedure is applicable for widespread pain affecting the limbs or trunk, and if need be it can be applied bilaterally. Not only will it relieve the same types of pain as are relieved by the various procedures already considered, but in my experience it will also generally relieve such otherwise intractable conditions as pain in a phantom limb, the lightening pains of tabes, and post-herpetic neuralgia. I should add that since Russell (1949) brought out his report, we have treated our cases of painful phantom limb by repeated percussion of the stump neuromas with encouraging results. The only cases of painful phantom limbs which we have submitted to chordotomy since then are those in which the neuromas are inaccessible.

The anatomical basis of anterolateral chordotomy is that it divides the lateral spino-thalamic tract and, consequently, the operation has to be performed on the side of the cord opposite to the side of the body which has to be denervated. Other tracts in the anterolateral columns are divided as well, but these do not seem to matter much. Sweating, for instance, is diminished over the body on the side of operation, while an ipsilateral Horner's syndrome follows a cervical chordotomy. A unilateral chordotomy does not disturb the sexual functions, but a bilateral operation causes loss of potentia in the male and of orgasm in the female.

The operation is usually performed in the upper thoracic region, for at this level the spinothalamic tract is usually better defined than at lower levels. A laminectomy of one or two 
vertebrae (e.g. $\mathrm{T}_{2}$ and 3 ) is sufficient to expose the cord. Next, one or more slips of the ligamentum denticulatum are divided so as to mobilize the cord. The chordotomy knife is then inserted transversely into the cord immediately in front of the line of the ligamentum denticulatum to a depth of $4 \mathrm{~mm}$., and is directed forwards so that its point emerges on the surface 2 or $3 \mathrm{~mm}$. anterior to the line of the anterior roots. Generally this incision has to be deepened by several small cuts, for the arachnoid is so tough that it cannot be incised satisfactorily in one stroke. Some surgeons rotate the cord through nearly a right angle before incising it, but by using a rightangled knife the cord can be satisfactorily incised with but little preliminary rotation. (A fragment of a safety razor blade set at right angles in the tip of an artery forceps makes a good knife.) Again, some surgeons perform the operation under local analgesia, for the actual incision of the cord is painless, and they can then check the sensory loss and extend the chordotomy if necessary (White et al., 1950). However, this is not essential, and results equally as satisfactory can be achieved under general anaesthesia, provided an adequate incision is made in the cord. Kahn and Peet (1948) and White et al. (1950) have rightly stressed that the incision in the anterolateral column of the cord must be deep and extensive; only the most anterior part of the column need be spared because of the proximity of the anterior spinal artery and because nerve pathways affecting sphincteric control are collected here.

Generally when the cord is incised beneath the second or third thoracic lamina, pain and thermal sensibility are completely abolished down the opposite side of the body below the costal margin. This suffices for painful conditions affecting the leg, pelvis or abdomen, provided the pain is unilateral. If, however, the pain affects both sides, a second incision can be made in the other side of the cord, but at a level one segment higher or lower. Convalescence following these operations is usually smooth, apart from a transient difficulty with the sphincters. Difficulty in initiating micturition and in emptying the bladder is quite often seen, especially after bilateral chordotomy, and may necessitate catheterization for a while. It generally clears up within a few days. Root pain due to trauma to a posterior root at the level of cord incision is occasionally encountered, and can be corrected by re-opening the laminectomy and dividing the posterior root.

For painful conditions of the upper limb or of the upper part of the thorax, high cervical chordotomy is necessary. The incision is usually made at a level between the points of emergence of the $\mathrm{C}_{1}$ and $\mathrm{C}_{2}$ nerves, and should always be performed under local analgesia so that the level of sensory loss produced can be tested after each cut as the incision in the cord is deepened. Since Lindsay and I (1945) reported our two cases of painful phantom of the arm which were relieved by high cervical chordotomy, I have performed the operation in four more cases. In each instance it was possible to produce a level of analgesia as high as the $C_{4}$ and $C_{5}$ segments, and all but one of the six patients have been benefited. The first of my patients has now been followed for seven years and still remains free of pain.

A major difficulty with both cervical and thoracic chordotomy has been to produce a permanent level of complete analgesia. In many of my cases some return of pain sensibility has occurred after several months, leading either to re-appearance of the original pain or to the appearance of perverted sensations in the lower part of the body. Two of my high cervical chordotomies have developed irritable paraesthesiae of the lower limb and trunk, although they remained free of pain in the phantom. In neither instance have these new sensations been as disturbing as the old, although they have been disturbing enough. In two thoracic chordotomies I was chagrined to find, within two months of the operation, a marked return to sensibility to pain.in the lower extremity with simultaneously a return of the old pain. In each instance a second chordotomy was made at a neighbouring level, but again pain sensibility returned after an interval. Occasionally it does not seem possible to produce a satisfactory and permanent loss of pain sensibility in spite of what seems an adequate incision in the cord. Possibly. the explanation may be bound up with anomalies of disturbance of the pain-carrying fibres in the cord, and that such anomalies do exist is well illustrated in the case reported by French and Peyton (1948) in which, following a unilateral upper choracic chordotomy, pain sensibility was lost down the same side of the patient's body.

\section{Spino-thalamic Tractotomy in the Brain Stem- Medullary Tractotomy and Mesencephalic Tracto- tomy}

It was because of the difficulty in relieving pain in the shoulders, neck and pectoral regions that section of the spino-thalamic tract was devised in the medulla by Schwartz and O'Leary (I94I)'and in the mid-brain by Walker (1942). The technical difficulties with these procedures are great, and no one has yet reported an experience of many cases. Moreover, in most instances only a partial or patchy analgesia has been obtained. I, myself, have not had occasion to try these procedures, but have relied on high cervical chordotomy for the relief of pain in the upper limb and pectoral 
regions. When pain has extended into the neck, I have performed this procedure on the contralateral side of the cord in combination with posterior rhizotomy of $\mathrm{C}_{2}, \mathrm{C}_{3}$ and, perhaps, $\mathrm{C}_{4}$, performed on the ipsilateral side of the cord.

\section{Excision of Sensory Cortex-Post-central Gyrec tomy}

In I944, de Gutiérrez-Mahoney reported a case of painful phantom of the upper limb, which he treated by resecting the arm area of the sensory cortex in the post-central gyrus of the opposite cerebral hemisphere. The phantom disappeared as well as the pain. Since then other clinicians have recorded their experiences, some reporting improvement (Echols and Colclough, 1947; Stone, 1950) and some failure (Horrax, I946; Trumble, 1948). In 1948, de Gutiérrez-Mahoney made a further report in which he outlined follow-up studies after the operation in four patients with painful phantom limb; three patients had persisting or recurrent symptoms. Horrax (1946) described two cases in which pain resulting from vascular or neoplastic disease of the central nervous system had been relieved by this procedure. A likely explanation of the failure with this operation is that the appreciation of pain in consciousness is not limited to the post-central gyrus, but can continue in secondary sensory areas.

\section{Procedures for Relief of Pain Arising in the Head}

The operative procedures available for the limbs and trunk have their counterparts in the operative procedures available for the head. These latter will be considered as follows:

\section{Section of Peripheral Branches of Cranial Nerves-Cranial Neurectomy}

The nerves principally concerned are the trigeminal nerve and the glossopharyngeal nerve. Avulsion of the infra-orbital branch of the trigeminal nerve or of its supratrochlear and supraorbital branches is sometimes performed for tic douloureux affecting their zones of distribution. Such simple measures are principally of value in frail elderly patients. In more active subjects a more radical procedure, such as section of the sensory root, is preferable, because the pain so frequently spreads to involve other branches of the division which are not denervated, and because regeneration of the divided nerves so commonly occurs, even when measures like plugging the infra-orbital canal are taken to prevent this.

Division of the glossopharyngeal nerve in the neck will relieve tic douloureux affecting that nerve. The approach to a neurosurgeon is more troublesome than by the intracranial route.
Furthermore there are risks of regeneration of the nerve. The approach consequently is seldom employed.

\section{Section of the Sensory Root of the Cranial Nerve-Sensory Root Section}

This type of procedure is analogous to posterior rhizotomy of a spinal nerve, and as with the latter there is no possibility: of regeneration afterwards. Partial or subtotal section of the sensory root of the trigeminal nerve is the standard neurosurgical procedure for trigeminal neuralgia, involving either the second or third division of the nerve, as it so commonly does. Only the lower and outer threequarters or four-fifths of the root are divided, thereby preserving sensibility in the forehead and cornea so that there is no risk of neuropathic keratitis. It is usually possible to preserve the motor root and so avoid a masticatory paralysis. The operation has generally been carried out by the classical approach of Frazier, which is a wholly extradural one, the gasserian ganglion being exposed by elevating the dura off the floor of the middle cranial fossa, atter the scalp and temporal muscle have been incised and a small opening made in the skull in the temporal fossa. This approach, however, is sometimes complicated by a facial palsy (due to traction on the great superficial petrosal nerve). Consequently many British neurosurgeons now practice an intradural approach, in which after opening the skull, they incise the dura and elevate the temporal lobe off the base of the skull, finally opening into Meckel's cave from above. This intradural approach also avoids the bleeding from venous sinuses which may render the extradural approach difficult.

For the intrequent case of trigeminal neuralgia which involves the first division, some surgeons still perform total section of the sensory root, while others resort to alcohol injection of the Gasserian ganglion, which has much the same effect but is simpler to perform. In my opinion, however, section of the descending root of the trigeminal nerve within the medulla is preferable to either of these procedures for pain involving the upper part of the face.

Section of the sensory root of the glossopharyngeal nerve lateral to the brain stem and before it enters the jugular foramen is the standard neurosurgical procedure for glossopharyngeal neuralgia. The approach is an intracranial one, much as for an eighth-nerve section.

\section{Alcohol Injection of the Gasserian Ganglion and its Branches}

Injection of certain peripheral branches of the trigeminal nerve with 90 per cent. alcohol-the third division at the foramen ovale, the infra- 
orbital nerve in its canal and the second division in the sphenomaxillary fossa-are commonly performed for trigeminal neuralgia affecting these branches. The techniques of injection are well described by Harris (1937). The numbness produced by these injections, however, is only temporary, persisting three to four months in the instance of the infra-orbital nerve and up to nine months for the third division. Re-appearance of pain is likely once cutaneous sensibility returns, and although alcohol injections may then be repeated, the common experience is that each subsequent injection becomes increasingly difficult and its effect less lasting. However, many neurosurgeons feel that it is well to employ one of these injections as the first stage in treatment of trigeminal neuralgia. For, once their old pain is forgotten, many patients complain bitterly of the numbness of their face, and a trial of the transient numbness which follows alcohol injection will condition must patients to accept the permanent numbness which follows operation.

In contrast to the transient effects of injection of its peripheral branches, alcohol injection of the Gasserian ganglion usually produces permanent numbness and consequently permanent relief of pain. However, if the cornea is also rendered anaesthetic, as so commonly happens, there is an ever present risk of neuropathic keratitis. Certainly this risk can be obviated by prescribing closely-fitting goggles or else a contact lens, which the patient must wear whenever he goes out of doors, and consequently alcohol injection of the ganglion is widely practised in this country. Moreover, alcohol injection of the ganglion is usually easy to perform, but against this must be balanced the extensive numbness of the whole face which it produces and the risk of corneal complications. My personal view is that when pain is confined to the second and third divisions it is preferable to perform a partial section of the sensory root, and when pain involves the first division to perform instead an intramedullary trigeminal tractotomy.

\section{Intramedullary Trigeminal Tractotomy}

Section of the descending tract of the trigeminal nerve within the medulla leads to loss of pain and thermal sensibility, but with sparing of touch. In my opinion it is the most satisfactory method of treating trigeminal neuralgia involving the upper part of the face or cases of trigeminal neuralgia in which an operation becomes necessary on the second side. The procedure is not an analogous one to a chordotomy, for it involves the primary and not the secondary relay of neurones, and produces its sensory loss over the same side of the face and not on the opposite side.
I have recently discussed the procedure in some detail (1949). It is an operative procedure which requires all the facilities of a specialized neurosurgical unit, and which is best carried out under local anaesthesia so that the depth and extent of the medullary incision can be controlled by sensory testing. Since Sjöqvist (1938) devised the operation its steps have been modified, and the descending tract is now usually divided at a level approximately $4 \mathrm{~mm}$. below the lower end of the fourth ventricle. At this level the tract is superficial in the medulla, forming with its nucleus the tuber cinereum of anatomists. With experience it is readily possible by this procedure to produce complete analgesia and thermalgesia of the upper two-thirds of the face, and often indeed of the whole face, without producing ataxia of the limbs or other troublesome side-effects. The third division is the most difficult to influence, because its fibres begin to leave the tract at a higher level in the brain stem. The principal advantage of the procedure is that, as touch is spared, the corneal reflex is retained, although it is usually diminished. There is thus no risk of neuropathic keratitis and the patient has no need for goggles or other precautions. Often the patient is not aware of any subjective disturbance of sensation in his face unless he is being tested. This contrasts with the constant numbness which many patients remark after injection of the Gasserian ganglion or sensory root section.

In my experience this operation has invariably led to permanent relief of trigeminal neuralgia of the tic douleureux type affecting the upper part of the face. It will not relieve the pain of postherpetic neuralgia, and neither will alcohol injection of the Gasserian ganglion nor section of its sensory root.

\section{Role of Prefrontal Leucotomy and Allied Procedures}

In 1946, Freeman and Watts reported that severe pain of organic disease could be relieved by prefrontal leucotomy. Since then others have confirmed their observations. The general experience following operation is that the patient still feels pain, although he no longer complains about it or lets it worry him, as he formerly did (Falconer, 1947). Although there may be no intellectual disturbance, there is, however, some blunting of the emotions following this operation, which consequently should be reserved for patients that are desperate with pain, cannot be relieved by a section of the pain pathways at an appropriate level and have a sufficient life expectancy to justify the operation and the period of post-operative confusion which goes with it. Prefrontal leucotomy as a therapeutic measure is an admission of failure 
and should be undertaken only when other measures are known to be ineffectual or have been tried unsuccessfully. It is usually not justified in such conditions as pelvic carcinoma, painful phantom limbs, causalgia and gastric crises, which can generally be relieved by an adequate chordotomy or sympathectomy, but it should be seriously considered for such conditions as intractable post-herpetic neuralgia of the face, and pain caused by vascular and other disturbances of the central nervous system which are not likely to respond to more orthodox procedures.

Further experience may show that it is possible to achieve the same degree of pain relief by a less extensive procedure. Le Beau (1950) has recorded his experience of bilateral topectomy for persistent pain due to various causes. In this procedure he excised a large part of the superior frontal convolutions on either side, and he claimed good results in a large proportion of his cases with a minimal degree of mental impairment. Scarff (1950) found that, following a unilateral prefrontal leucotomy in which the connections of the frontal lobe were thoroughly transected, pain was relieved with little or no psychical clouding. It was immaterial which cerebral hemisphere was operated on. My experience, like that of others, is that after a short interval pain may again become troublesome, necessitating a leucotomy of the opposite frontal lobe. There is, however, no contraindication to performing frontal leucotomies in stages, and White and his colleagues (1950) favour a complete unilateral prefrontal leucotomy in the first instance, followed at a later date by a section of the other cerebral hemisphere, if the pain returns or is inadequately relieved. Their experience is that by this stage procedure pain is relieved as effectively and with less psychical deterioration than after the one-stage bilateral operation.

\section{Further Comments}

It is, in some respects, a pity that inoperable neoplastic disease bulks so largely as a cause of intractable pain, for in the eyes of many clinicians the surgical relief of pain is but a means of easing the last days of a patient who will shortly succumb from a malignant lesion. Yet there are several conditions, in the aggregate perhaps as numerous as cases of pain due to malignant disease, which are not lethal in themselves and which yet cause pain of sufficient severity to justify surgical intervention. Examples are seen in tic douloureux and other intractable neuralgias, in causalgia and painful phantom limbs, and in the involvement of nerve structures by scar tissue. It is essential that the operative procedures undertaken in such conditions should be effective and long-lasting, and yet safe and without militating side-effects. One should deprecate too ready a tendency to submit patients to leucotomy without deciding whether the pain is of organic or psychical origin, and whether it can be relieved by some less disturbing procedure.

The use of diagnostic procaine blocks of the pain pathways is often of great assistance both in diagnosis and in deciding what surgical procedure should be tried in a particular patient.

Surgical treatment offers its most certain prospects for relieving pain when it is of somatic or visceral origin. It is otherwise with pain due to organic disease of the central nervous system. I have indicated our comparative helplessness in treating post-herpetic neuralgia, the 'thalamic syndrome,' and other conditions affecting the nervous system, except by the drastic procedure of prefrontal leucotomy. Even the common condition of migraine still challenges us. We have still a great deal to learn.

\section{BIBLIOGRAPHY}

LE BEAU, J. (1950), $\mathcal{F}$. Neurosurg., 7, 79.

BRODAL, A. (1947), Arch. Neurol. Psychiat., Chicago, 57, 292.

BRODAL, A. (1948), ' Neurological anatomy in relation to clinical medicine,' Oxford.

ECHOLS, D. H., and COLCLOUGH, J. A. (1947), f. Amer. med. Ass., 134,1476 .

FALCONER, M. A. (1947), Proc. Assoc. Res. nerv. ment. Dis., 27, 706.

FALCONER, M. A. (1949), F. Neurol. Neurosurg. Psychiat., 12, 297. FALCONER, M. A., and LINDSAY, J. S. B. (1945), Brit. $\mathcal{f}$. Surg., 33, 301.

FREEMAN, W., and WATTS, J. W. (1946), Lancet, i, 953.

FRENCH, L. A., and PEYTON, W. T. (1948), F. Neurosurg., 5,403 .

FULTON, J. F. (1943), 'Physiology of the nervous system,' New York.

DE GUTIERREZ-MAHONEY, C. G. (1944), Ibid., I, 156.

DE GUTIERREZ-MAHONEY, C. G. (1948), Surg. Clin. $N$. Amer., 00, 481.

HARRIS, W. (1937), 'The facial neuralgias,' London.

HORRAX, G. (1946), Surgery, 20, 593.

INGERSOLL, F. M., and MEIGS, J. V. (1948), Nerv Engl. $f$. Med., 238, 357.
KAHN, E. A., and PEET, M. M. (1948), F. Neurosurg., 5, 276. LINDGREN, I., and OLIVECRONA, H. (1947), $\mathcal{f}$. Neurosurg., $4,19$.

LIVINGSTON, W. K. (1943), 'Pain mechanisms,' New York. MACFARLANE, W. V. (1949), Aus. N.Z.F. Surg., 18, 191. RUSSELL, W. R. (1936), Lancet, i, 595.

RUSSELL, W. R. (1949), Brit. med. f., i, 1024.

SCARFF, J. E. (1950), $\mathcal{\exists}$.' Neurosurg., 7, 330.

SCHWARTZ, H. G., and O'LEARY, J. L. (194I), Surgery, 9, 183. SJOQVIST, O. (1938), Acta psychiat. neurol., Suppl. 17.

STONE, T. T. (1950), Arch. Neurol. Psychiat., 63, 739.

TRUMBLE, H. C., quoted by SUNDERLAND, S., and KELLY, M. (1948), Aust. N.Z.F. Siurg., 18, 75.

ULMER, J. L., and MAYFIELD, F. H. (1946), Surg. Gynec. Obstet., 83, 789 .

WALKER, A. E. (1942), Arch. Neurol. Psychiat., 48, 865.

WHITE, J. C. (1946), Amer. F. Surg., 72, 468.

WHITE, J. C. (1950), Lancet, ii, I6I.

WHITE, J. C., and BLAND, E. F. (1948), Medicine, 27, 1 .

WHITE, J. C., SWEET, W. H., HAWKINS, R., and NILGES, R. G. (1950), Brain, 73, 346. 